\title{
ON MÖBIUS' INVERSION FORMULA AND CLOSED SETS OF FUNCTIONS
}

\author{
BY \\ OTTO SZÁSZ(')
}

1. Introduction. We consider the functions $x(t)$ of a variable $t$, defined on a point set $E$, consisting of an infinity of real or complex numbers. We assume that each function is bounded, and denote the totality of these functions by $S$. Given a sequence of real or complex numbers:

$$
\begin{aligned}
& \lambda_{1}=1, \lambda_{2}, \lambda_{3}, \cdots, \lambda_{v} \neq \lambda_{u} \\
& \lambda_{v} \neq 0
\end{aligned}
$$

for $v \neq u$,

for all $v$,

and a sequence of real or complex numbers

$$
a_{1}=1, a_{2}, a_{3}, \cdots, \quad \sum\left|a_{n}\right|<\infty,
$$

we now assume that if $t \in E$, then all $\lambda_{n} t \in E$.

Consider the transformation

$$
y(t)=\sum_{1}^{\infty} a_{n} x\left(\lambda_{n} t\right)=x(t)+\sum_{2}^{\infty} a_{n} x\left(\lambda_{n} t\right)=I(x)+A(x),
$$

say; clearly, if $x(t) \in S$, then $y(t) \in S$. We further assume that the sequence (1.1) has the property: (P) the product of any two $\lambda$ is a $\lambda$. It then follows that $\lambda_{v_{1}}^{\alpha_{1}} \cdots \lambda_{p_{k}}^{\alpha_{k}}$ is in the sequence $(1.1) ; \alpha_{1}, \alpha_{2}, \cdots$ are non-negative integers. We shall give conditions under which the functional equation (1.2) has a unique inverse; we shall express the inverse in the form $x(t)=\sum b_{n} y\left(\lambda_{n} t\right)$, and we shall give applications to problems of closure and completeness. The special case $\lambda_{n}=n$ is a generalization of an inversion formula due to Möbius. It has been applied to prove completeness (closure) of certain sequences in two papers by E. Hille and the author [5, I and II].

Actually the assumption $(P)$ is no restriction of generality; for adjoining successively all $\lambda$-products which are missing in (1.1), we get a new denumerable $\lambda^{\prime}$-set, and (1.2) can be rewritten in the form $\sum a_{n}^{\prime} x\left(\lambda_{n}^{\prime} t\right)$; we must however also extend the point set $E$, so that it contains all points $\lambda_{n}^{\prime} t$ of $t \in E^{\prime}$.

Using the terminology of abstract spaces (see $[1$, pp. 11, 53]), defining

Presented to the Society, August 23, 1946; received by the editors June 3, 1946.

(1) The main results of this paper were presented in December, 1944, at Brown University, at the University of Pennsylvania, and the Ohio State University. In the meantime closure theorems of a similar type have been announced by D. G. Bourgin [2]. Numbers in brackets refer to the literature at the end of the paper. 
distance of two elements $x_{1}(t), x_{2}(t)$ by $\left(x_{1}, x_{2}\right)=1$.u.b.t $t \in E\left|x_{1}(t)-x_{2}(t)\right|$, and putting $\|x(t)\|=(x(t), 0)$, we see that $S$ is a normed vector space of type (B). Under the assumption (1.1a), (1.2) is a linear operation in $S$ and

$$
\|A(x)\| \leqq\|x\| \sum_{2}^{\infty}\left|a_{n}\right| .
$$

\section{The inverse transformation.}

THEOREM 1. If $\sum_{2}^{\infty}\left|a_{n}\right|<1$, then the transformation (1.2) has a unique inverse

$$
x(t)=y(t)+B(y),
$$

and $B(y)$ is again of the form

$$
B(y)=\sum_{2}^{\infty} b_{n} y\left(\lambda_{n} t\right), \quad \sum\left|b_{n}\right|<\infty .
$$

Formal operational calculus $\left({ }^{2}\right)$ yields from (1.2)

$$
x(t)=(I+A)^{-1}(y)=\left(\sum_{0}^{\infty}(-1)^{k} A^{k}\right)(y)=\sum_{0}^{\infty}(-1)^{k} A^{k}(y),
$$

where $A^{0}(y)=I(y)=y$, and

$$
A^{k}(y)=\sum_{v_{1}=2}^{\infty} \cdots \sum_{v_{k}=2}^{\infty} a_{v_{1}} \cdots a_{v_{k}} y\left(\lambda_{v_{1}} \cdots \lambda_{v_{k}} t\right), \quad k \geqq 1 .
$$

Let

$$
\sum_{2}^{\infty}\left|a_{n}\right|=\bar{a}<1, \quad \underset{i \in E}{\text { l.u.b }}|y(t)|=\eta,
$$

then the general term in the multiple series (2.4) is majorized by $\left|a_{v_{1}} \cdots a_{v_{k}}\right| \eta$, hence the series (2.4) is absolutely and uniformly convergent ${ }^{\circ}$ for $t \in \epsilon$, and

$$
\left|A^{k}(y)\right| \leqq \eta\left(\sum_{2}^{\infty}\left|a_{n}\right|\right)^{k}=\eta \bar{a}^{k}
$$

Substituting (2.4) into (2.3) we get again an absolutely and uniformly convergent multiple series, representing a function $x(t) \in S$; moreover

$$
|x(t)| \leqq \eta \sum_{0}^{\infty} \bar{a}^{k}=\frac{\eta}{1-\bar{a}} .
$$

We next show that $x(t)$ satisfies the equation (1.2); let

(2) I am indebted to E. R. Lorch for reminding me of this procedure. 


$$
x_{n}(t)=\sum_{k=0}^{n}(-1)^{k} A^{k}(y),
$$

then evidently

$$
x_{n}(t) \rightarrow x(t) \text { uniformly in } E
$$

Furthermore

$$
\begin{aligned}
(I+A) x_{n} & =\sum_{0}^{n}(-1)^{k} A^{k}(y)+\sum_{0}^{n}(-1)^{k} A^{k+1}(y) \\
& =\left(I+(-1)^{n} A^{n+1}\right) y \rightarrow I(y),
\end{aligned}
$$

as $n \rightarrow \infty$; this proves $(I+A) x=y$.

To prove that (2.1) is the only solution of (1.2), we show that

$$
(I+A) x=0
$$

has only the solution $x=0$. In fact if

$$
A(x)=-I(x)=-x,
$$

then

$$
\underset{i \in E}{\text { l.u.b. }}|x(t)| \leqq \bar{a} \text { l.u.b. }|x(t)| \text {; }
$$

but $\bar{a}<1$, hence $x=0$ for all $t \in E$.

Finally collecting corresponding terms in (2.3), (2.4), we get (2.2), where

$$
b_{n}=-a_{n}+\sum a_{v_{1}} a_{v_{2}}-\cdots+(-1)^{k} \sum a_{v_{1}}^{\alpha_{1}} a_{v_{2}}^{\alpha_{2}} \cdots+\cdots
$$

$$
=\sum_{k=1}^{\infty}(-1)^{k} B_{k}(n) \text {, }
$$

say; to each factorization $\lambda_{n}=\lambda_{v_{1}}^{\alpha_{1}} \lambda_{z_{2}}^{\alpha_{2}} \cdots, \alpha_{1}+\alpha_{2}+\cdots=k$, into $k$ not necessarily different factors, $\lambda_{v}>1$, corresponds a term in $B_{k}(n)$, the order of different factors $\lambda_{0}^{\alpha}$ being essential. In other words, we have the formal identity

$$
\left(\sum_{2}^{\infty} \lambda_{v}^{-s}\right)^{k}=\sum_{v=2}^{\infty} C_{k}(v) \lambda_{v}^{-s}
$$

where $C_{k}(n)$ is the number of terms in $B_{k}(n)$. It is clear that

$$
\sum_{2}^{\infty}\left|b_{n}\right| \leqq \sum_{1}^{\infty} \bar{a}^{k}=\frac{\bar{a}}{1-\bar{a}}
$$

and equality holds if all $a_{n}$ are not greater than 0 .

While the solution (2.1) is unique, it is possible that the representation (2.2) is not unique; this is the case if and only if 0 has a development 


$$
0=\sum_{2}^{\infty} \beta_{n} y\left(\lambda_{n} t\right), \quad \sum\left|\beta_{n}\right|<\infty,
$$

and not all $\beta_{n}=0$.

3. Other conditions for the inversion of the functional equation.

THEOREM 2. Suppose that $\sum_{n, 0-1}^{\infty}\left|a_{n} u_{v} y\left(\lambda_{n} \lambda_{v} t\right)\right|<\infty$, where $u_{v}$ is a solution of the system of equations

$$
\begin{gathered}
a_{1}=1, \quad u_{1}=1, \\
\sum u_{v} a_{n}=0, \text { summing over all } \lambda_{v} \lambda_{n}=\lambda_{m}, m \geqq 2,
\end{gathered}
$$

then

$$
x(t)=\sum_{1}^{\infty} u_{n} y\left(\lambda_{n} t\right)
$$

is a solution of the equation (1.2). Furthermore any solution of (1.2) which satisfies the condition

$$
\sum_{n, v}\left|a_{n} u_{0} x\left(\lambda_{n} \lambda_{v} t\right)\right|<\infty,
$$

where $u_{v}$ is a solution of (3.1), is represented by (3.2).

Proof. Under the assumption

$$
\sum_{n, v}\left|a_{n} u_{v} y\left(\lambda_{n} \lambda_{v} t\right)\right|<\infty
$$

substitution of (3.2) into (1.2) yields, in view of (3.1)

$$
\sum_{n=1}^{\infty} a_{n}\left(\sum_{v=1}^{\infty} u_{v} y\left(\lambda_{n} \lambda_{v} t\right)\right)=\sum_{m=1}^{\infty}\left(\sum_{\lambda_{v} \lambda_{n}=\lambda_{m}} u_{v} a_{n}\right) y\left(\lambda_{m} t\right)=y(t) .
$$

This proves the first part of our theorem. Furthermore if $x(t)$ is a solution of (1.2) satisfying (3.3), then from

$$
y\left(\lambda_{v} t\right)=\sum_{n=1}^{\infty} a_{n} x\left(\lambda_{n} \lambda_{v} t\right)
$$

and from (3.1),

$$
\sum_{1}^{\infty} u_{v} y\left(\lambda_{v} t\right)=\sum_{v, n} a_{n} u_{v} x\left(\lambda_{n} \lambda_{v} t\right)=x(t) .
$$

This proves the second part of Theorem 2. For $\lambda_{n}=n$ see $[4$, Theorem 3.2.1; 9, chap. 3].

Note that we no longer assume that $x(t), y(t)$ belong to $S$. The theorem holds for those values of $t$ which satisfy the assumption of absolute con- 
vergence of (3.3) and (3.4). In Theorem 1, we had the assumptions $\sum_{2}^{\infty}\left|a_{n}\right|<1$, $x(t), y(t)$ bounded in $E$. The unique solution was

$$
x(t)=\sum_{1}^{\infty} b_{n} y\left(\lambda_{n} t\right)
$$

where $b_{n}$ is defined by (2.5), and $\sum\left|b_{n}\right|<\infty$. We can now show that $b_{n}$ is a solution of (3.1). Clearly (3.1) is equivalent to the formal identity

$$
\left(\sum_{1}^{\infty} a_{n} \lambda_{n}^{-8}\right)\left(\sum_{1}^{\infty} u_{n} \lambda_{n}^{-8}\right)=1 \text {, }
$$

if we employ Dirichlet-multiplication of the two series. If we choose $y(t)=t^{-\bullet}$ $t \geqq 1$, (3.5) becomes

$$
x(t)=t^{-*} \sum_{1}^{\infty} b_{n} \lambda_{n}^{-*},
$$

while equation (1.2) becomes

$$
\vec{t}^{-s}=\sum_{n, v=1}^{\infty} a_{n} b_{v}\left(\lambda_{n} \lambda_{v}\right)^{-s} \overrightarrow{t^{-s}}=t^{-s}\left(\sum a_{n} \lambda_{n}^{-s}\right)\left(\sum b_{v} \lambda_{v}^{-s}\right) ;
$$

thus (3.6) holds, which proves (3.1) with $u_{n}=b_{n}$.

It follows from Theorem 2 that if (3.1) has exactly one solution which satisfies (3.4), then the equation (1.2) has exactly one solution which satisfies (3.3), and the solution is given by (3.2).

We call a sequence $u_{v}$ that satisfies (3.1) a reciprocal or inverse of the sequence $a_{n}$ of $\lambda$-type.

For $\lambda_{n}=n,(3.1)$ and (3.2) reduce to the inversion formulae of Möbius: $a_{1}=1, u_{1}=1$,

$$
\begin{gathered}
\sum_{v n=m} u_{v} a_{n}=0, \quad m>1, \quad \text { or }\left(\sum a_{n} n^{-s}\right)\left(\sum u_{n} n^{-\triangleleft}\right)=1, \\
x(t)=\sum u_{n} y(n t) .
\end{gathered}
$$

4. The inverse of certain sequences. We now assume that the sequences $a_{n}, \lambda_{n}$ are completely multiplicative, that is, $a_{1}=1, \lambda_{1}=1$,

$$
a_{n} a_{v}=a_{n v}, \quad \lambda_{n} \lambda_{v}=\lambda_{n v}, \quad n, v=1,23, \cdots
$$

A multiplicative sequence $a_{n}$ is uniquely determined by prescribing the subsequence $a_{p}, p$ any prime, arbitrarily. For if $n=p_{1}^{\alpha_{1}} p_{2}^{\alpha_{2}} \cdots$, then $a_{n}=a_{p_{1}}^{\alpha_{1}} a_{p_{2}}^{\alpha_{2}} \cdots$. Furthermore if $\sum\left|a_{n}\right|<\infty$, then $a_{p \alpha}=a_{p}^{\alpha} \rightarrow 0$ as $\alpha \rightarrow \infty$, hence $\left|a_{p}\right|<1$, and $\left|a_{n}\right|<1, n>1$. Moreover

$$
\prod_{p} \frac{1}{1-\left|a_{p}\right|}=\prod_{p} \sum_{\alpha}\left|a_{p}\right|^{\alpha}=\sum\left|a_{n}\right|,
$$


hence $\sum\left|a_{n}\right|<\infty$ if and only if $\left|a_{p}\right|<1$ and if $\sum\left|a_{p}\right|<\infty$.

If $(4.1)$ holds then formula $(2.5)$ becomes

$$
b_{n}=-a_{n}+C_{2}(n) a_{n}-\cdots=a_{n} \sum_{k=1}^{\infty}(-1)^{k} C_{k}(n)
$$

where $C_{k}(n)$ is the number of factorizations of $n$,

$$
n=v_{1}^{\alpha_{1}} v_{2}^{\alpha_{2}} \ldots, \quad \alpha_{1}+\alpha_{2}+\cdots=k,
$$

into $k$ not necessarily different factors greater than 1 , the order of different factors being essential. (For example, $12=2^{2} \cdot 3=3 \cdot 2^{2}=2 \cdot 3 \cdot 2$ are the different factorizations of 12.) The inverse is now indrpendent of the sequence $\lambda_{n}$. The series $\sum_{k}(-1)^{k} C_{k}(n)$ is actually finite as $C_{k}(n)=0$ for $k>n . \sum_{k} C_{k}(n)$ $=F(n)$ is the number of factorizations of $n$ into factors greater than one, order being essential. It is clear that

$$
\sum_{n=2}^{\infty} C_{k}(n) n^{-s}=(\zeta(s)-1)^{k}, \quad R s>1, \quad k=1,2,3, \cdots,
$$

and, putting $F(1)=1$,

$$
\sum_{1}^{\infty} F(n) n^{-s}=(2-\zeta(s))^{-1} ; \quad \zeta(s)=\sum_{1}^{\infty} n^{-s}
$$

From (4.3)

$$
\sum_{k=1}^{\infty}(-1)^{k} \sum_{n=2}^{\infty} C_{k}(n) n^{-s}=\sum_{1}^{\infty}(1-\zeta(s))^{k}=\frac{1-\zeta(s)}{\zeta(s)},
$$

or

$$
\sum_{n=2}^{\infty}\left(\sum_{k=1}^{\infty}(-1)^{k} C_{k}(n)\right) n^{-s}=\frac{1}{\zeta(s)}-1=\sum_{2}^{\infty} \mu(n) n^{-s}
$$

hence

$$
\sum_{k=1}^{\infty}(-1){ }^{k} C_{k}(n)=\mu(n),
$$

and

$$
b_{n}=\mu(n) a_{n} .
$$

$\mu(n)$ is the Möbius' function: $\mu(n)=0$, if $n$ contains a quadratic factor, $\mu(n)=(-1)^{k}$, if $n$ is the product of $k$ different primes.

The system (3.1) now reduces to (3.7), which has the unique solution $u_{n}=\mu(n) a_{n}=b_{n}$. This is seen from the formal identities 


$$
\sum_{1}^{\infty} a_{n} n^{-s}=\prod_{p}\left(1-a_{p} p^{-s}\right)^{-1}, \quad \prod_{p}\left(1-a_{p} p^{-s}\right)=\sum \mu(n) a_{n} n^{-s} .
$$

Note. If the sequence $a_{n}$ is multiplicative and if $\sum\left|a_{n}\right|<\infty$, then evidently

$$
\left(\sum_{2}^{\infty}\left|a_{n}\right|\right)^{k}=\sum_{n=2}^{\infty} C_{k}(n)\left|a_{n}\right|<\infty \text {. }
$$

If in addition $\sum_{2}^{\infty}\left|a_{n}\right|<1$, then $\sum_{k=1}^{\infty}\left(\sum_{2}^{\infty}\left|a_{n}\right|\right)^{k}=\sum F(n)\left|a_{n}\right|$ is convergent.

$C_{2}(n)$ is closely related to $d(n)$, the number of divisors of $n$; we have

$$
\sum_{1}^{\infty} d(n) n^{-s}=(\zeta(s))^{2},
$$

which yields, in view of (4.3) with $k=2, C_{2}(n)=d(n)-2$. It follows that $\sum d(n)\left|a_{n}\right|<\infty$. We shall show that moreover

$$
\sum d^{2}(n)\left|a_{n}\right|<\infty \text {. }
$$

We employ the formula

$$
\zeta^{4}(s)=\zeta(2 s) \sum^{\infty} d^{2}(n) n^{-s}=\sum c_{n} n^{-\bullet}
$$

say; clearly

$$
c_{n} \geqq d^{2}(n) .
$$

Furthermore

$$
\sum c_{n} n^{-s}=(\zeta(s)-1)^{4}+4(\zeta(s)-1)^{8}+\cdots,
$$

from which it follows easily that

$$
d^{2}(n) \leqq c_{n}<15 C_{4}(n) .
$$

Now (4.4) with $k=4$ yields (4.5).

The conditions of Theorem 2 now become (as $u_{n}=\mu(n) a_{n}$ )

$$
\begin{aligned}
\sum_{n, v}\left|a_{n} u_{v} y\left(\lambda_{n} \lambda_{v} t\right)\right| & =\sum_{n, v}\left|\mu(v) a_{n v} y\left(\lambda_{n v} t\right)\right| \\
& =\sum_{m=1}^{\infty}\left(\sum_{\delta \mid m}|\mu(\delta)|\right)\left|a_{m} y\left(\lambda_{m} t\right)\right|<\infty,
\end{aligned}
$$

and

$$
\sum_{n, v}\left|a_{n} u_{v} x\left(\lambda_{n} \lambda_{r} t\right)\right|=\sum_{m}\left(\sum_{\delta \mid m}|\mu(\delta)|\right)\left|a_{m} x\left(\lambda_{m} t\right)\right|<\infty .
$$

We have thus the following theorem: 
THEOREM 3. If (4.1) holds, then the sequence $a_{n}$ has a unique reciprocal $u_{n}=\mu(n) a_{n}$. Moreover if (4.6) holds, then

$$
x(t)=\sum_{1}^{\infty} \mu(n) a_{n} y\left(\lambda_{n} t\right)
$$

is a solution of (1.2). There is at most one solution satisfying (4.7); if it exists, it is given by (4.8).

It follows from the second part of the theorem, that the only solution of the homogeneous equation $\sum a_{n} x\left(\lambda_{n} t\right)=0$ which satisfies (4.7) is $x(t)=0$. For an application see $\S 6$.

If in particular $\sum\left|a_{n}\right|<\infty$ and if $y(t)$ is bounded, then (4.6) holds (see the Note) hence (4.8) is the unique solution of (1.2) which satisfies (4.7).

5. Application to approximation and closure. Given a sequence of functions $\phi_{n}(t), n \geqq 1, t \in E$, we call the totality of functions which can be approximated uniformly by linear aggregates $\sum_{1}^{n} c_{v} \phi_{v}(t)$ in $E$, the span of the sequence $\left\{\phi_{n}(t)\right\}$, and denote it by $M\left(\phi_{n}\right)$. It is also called the closed linear manifold determined by the sequence $\phi_{n}(t)$.

Consider now the sequences $x\left(\lambda_{n} t\right)=x_{n}$, and $y\left(\lambda_{n} t\right)=y_{n}$; we shall prove:

Theorem 4. If the assumptions of Theorem 1 or Theorem 3 with uniform convergence are satisfied, then the sequences $x\left(\lambda_{n} t\right), y\left(\lambda_{n} t\right), n=1,2, \cdots$, define the same span.

(a) It is easy to see that any function $f(t)$ that can be approximated uniformly by the sequence $y\left(\lambda_{n} t\right)$, that is, $f(t) \in M\left(y_{n}\right)$, also belongs to $M\left(x_{n}\right)$. For let $\epsilon>0$ be given, and

$$
\left|f(t)-\sum_{1}^{n} c_{k} y\left(\lambda_{k} t\right)\right|<\epsilon, \quad t \in E, \quad c_{k}=c_{k}(n, \epsilon), \quad n=n(\epsilon) ;
$$

then from (1.2)

$$
\left|f(t)-\sum_{k=1}^{n} \sum_{v=1}^{\infty} a_{v} x\left(\lambda_{v} \lambda_{k} t\right)\right|<\epsilon .
$$

Furthermore, $n$ being fixed,

$$
\left|\sum_{k=1}^{n} c_{k} \sum_{v=m}^{\infty} a_{v} x\left(\lambda_{v} \lambda_{k} t\right)\right|<\epsilon \quad \text { for } m>m_{0}(\epsilon) .
$$

Thus $f(t)$ is approximated uniformly by linear aggregates of the functions $x\left(\lambda_{v} t\right)$. Hence $M\left(y_{n}\right) \subset M\left(x_{n}\right)$.

(b) The converse follows from the existence of an inverse of the form (2.2).

Example 1. Let 


$$
y(t)=\sum_{1}^{\infty}(2 v-1)^{-2}(1-\cos (2 v-1) \pi t)
$$

then

$$
y(t)=\left(\pi^{2} / 4\right)|t| \text { for }-1 \leqq t \leqq 1,
$$

and the funtional equations $y(-t)=y(t)=y(t+2)$ define $y(t)$ for all real $t$; $t=1 / 2$ yields

$$
\sum_{1}^{\infty}(2 v-1)^{-2}=\frac{\pi^{2}}{8}
$$

hence

$$
\sum_{2}^{\infty}(2 v-1)^{-2}=\frac{\pi^{2}}{8}-1<1 .
$$

Theorem 4 applies with $x(t)=1-\cos \pi t, a_{2 v-1}=(2 v-1)^{-2}, a_{2 v}=0$. Now $M\left(x_{n}\right)$ is the totality of all continuous functions in the interval $(0,1)$, vanishing at $t=0$. Hence the same is true of $M\left(y_{n}\right)$; here $x_{n}=x(n t), y_{n}=y(n t)$.

Example 2. Consider $|\sin t|=2 / \pi-(4 / \pi) \sum_{1}^{\infty} \cos 2 v t /\left(4 v^{2}-1\right)$, hence

$$
\sum_{1}^{\infty}\left(4 v^{2}-1\right)^{-1}=\frac{1}{2}, \quad \frac{3 \pi}{4}|\sin t|=3 \sum_{1}^{\infty} \frac{1-\cos v t}{4 v^{2}-1} .
$$

Put $x(t)=1-\cos 2 t, a_{v}=3\left(4 v^{2}-1\right)^{-1}$; then $\sum_{2}^{\infty} a_{v}=3 / 2-1=1 / 2<1$; again Theorem 4 applies. Now $M\left(x_{n}\right)$ is the totality of all continuous functions in $(0, \pi / 2)$, vanishing at $t=0$. The same is true for the sequence $y_{n}=|\sin n t|$. We have thus proved the theorem:

THEOREM 5. Either of the sequences $\phi(n t)$ and $|\sin (\pi n t / 2)|$ is a base of all continuous even functions in $(-1,+1)$, vanishing at $t=0$. Here $\phi(t)=|t|$ $=\phi(t+2),-1 \leqq t \leqq 1$.

A more general case is furnished by $\phi(t)=\sum_{1}^{\infty} a_{v}(1-\cos v t)$, where $a_{1}=1$ and $\sum_{2}^{\infty}\left|a_{n}\right|<1$, or $a_{n} a_{v}=a_{n v}$ and $\sum\left|a_{n}\right|<\infty$.

We are now in a position to discuss a question which arose in a conversation with $R$. P. Boas, Jr. Let $\alpha>0$ and

$$
\begin{aligned}
y(t) & =(\sin t)^{\alpha} & \text { for } 0 \leqq t \leqq \pi, \\
y(-t) & =-y(t)=y(2 \pi-t) . &
\end{aligned}
$$

$y(t)$ has a Fourier sine-series (if only $\alpha>-1$ )

$$
y(t) \sim \sum_{1}^{\infty} a_{n} \sin n t, \quad a_{n}=\frac{2}{\pi} \int_{0}^{\pi}(\sin t)^{\alpha} \sin n t d t=a_{n}^{(\alpha)} .
$$

The question is: for what values of $\alpha>0$ does the sequence $y(n t), n=1,2$, 
$3, \cdots$, span the same space as the sequence $\{\sin n t\}$ in $C(0, \pi)$ ?

We shall prove:

TheOREM. 6. Let $\alpha>1 / 3 ; y(t)=(\sin t)^{\alpha}$ for $0 \leqq t \leqq \pi ; y(-t)=-y(t)$ $=y(2 \pi-t)$. The span of the sequence $\{y(n t)\}$ is identical with the span of the sequence $\{\sin n t\}$ in $C(0, \pi)$.

On putting $a_{0}=0$, we have from (5.1) for $\alpha>-1, n \geqq 1$,

$$
\begin{aligned}
\frac{\pi}{2}\left(a_{n+1}+a_{n-1}\right) & =\int_{0}^{\pi}(\sin t)^{\alpha}\{\sin (n+1) t+\sin (n-1) t\} d t \\
& =2 \int_{0}^{\pi}(\sin t)^{\alpha} \sin n t \cos t d t \\
& =-\frac{2 n}{\alpha+1} \int_{0}^{\pi}(\sin t)^{\alpha+1} \cos n t d t
\end{aligned}
$$

and

$$
\begin{aligned}
2 \int_{0}^{\pi}(\sin t)^{\alpha+1} \cos n t d t & =\int_{0}^{\pi}(\sin t)^{\alpha}\{\sin (n+1) t-\sin (n-1) t\} d t \\
& =\frac{\pi}{2}\left(a_{n+1}-a_{n-1}\right)
\end{aligned}
$$

Thus

or

$$
(\alpha+1)\left(a_{n+1}+a_{n-1}\right)=n\left(a_{n-1}-a_{n+1}\right),
$$

$$
(n+\alpha+1) a_{n+1}=(n-\alpha-1) a_{n-1}, \quad n \geqq 1, \alpha>-1
$$

In particular

$$
\begin{array}{rlrl}
a_{2 v} & =0, & v=0,1,2, \cdots, \\
(2 v+\alpha+1) a_{2 v+1} & =(2 v-\alpha-1) a_{2 v-1}, & v & \geqq 1, \\
a_{2 n+1} & =a_{1} \prod_{v=1}^{n} \frac{2 v-\alpha-1}{2 v+\alpha+1}=O\left(n^{-1-\alpha}\right) & \text { as } n \rightarrow \infty .
\end{array}
$$

It follows that $\sum\left|a_{2 n+1}\right|<\infty$ for $\alpha>0$, and $0<a_{2 n+1}<a_{2 n-1}$ for $-1<\alpha<1$. For $n=1: a_{1}=(2 / \pi) \int_{0}^{\pi}(\sin t)^{\alpha+1} d t>0$. From (5.4)

$$
(2 v+\alpha+1) a_{2 v+1}=(2 v+\alpha-1) a_{2 v-1}-2 \alpha a_{2 v-1},
$$

hence

$$
(2 n+\alpha+1) a_{2 n+1}=(\alpha+1) a_{1}-2 \alpha \sum_{1}^{n} a_{2 v-1}, \quad 2 \alpha \sum_{1}^{\infty} a_{2 v-1}=(\alpha+1) a_{1}
$$


(from (5.5)). Let first $1 / 3<\alpha<1$; then $a_{2 n-1}>0, n>0$, and

$$
\sum_{2}^{\infty} a_{2 v-1}=\frac{1-\alpha}{2 \alpha} a_{1}<a_{1},
$$

hence Theorem 4 applies.

Next let $1<\alpha \leqq 3$; then $a_{2 n+1} \leqq 0$ for $n>0$, and

$$
\sum_{2}^{\infty}\left|a_{2 v-1}\right|=-\sum_{2}^{\infty} a_{2 v-1}=\frac{\alpha-1}{2 \alpha} a_{1}<a_{1} .
$$

Finally for $\alpha>3$ from (5.5)

$$
a_{2 n+1}^{(\alpha-2)}=a_{1}^{(\alpha-2)} \prod_{1}^{n} \frac{2 v-\alpha+1}{2 v+\alpha-1}
$$

and

$$
\frac{a_{2 n+1}^{(\alpha)}}{a_{1}^{(\alpha)}}=\frac{1-\alpha^{2}}{(2 n+\alpha-1)(2 n+\alpha+1)} \frac{a_{2 n+1}^{(\alpha-2)}}{a_{1}^{(\alpha-2)}} .
$$

Thus (5.6) holds for all $\alpha>1 / 3$, and our theorem follows from Theorem 4 . This proves Theorem 6 .

We next consider the function

$$
\begin{aligned}
\phi(t) & =(\sin t)^{\alpha}, & 0 \leqq t \leqq \pi, \alpha>0, \\
\phi(-t) & =\phi(t)=\phi(2 \pi-t) . &
\end{aligned}
$$

$\phi(t)$ has a Fourier cosine-series

$$
\phi(t) \sim \frac{1}{2} a_{0}^{*}+\sum_{1}^{\infty} a_{n}^{*} \cos n t, \quad a_{n}^{*}=\frac{2}{\pi} \int_{0}^{\pi}(\sin t)^{\alpha} \cos n t d t,
$$

hence, from (5.2) and (5.3),

$$
a_{n}^{*}=-\frac{\alpha+1}{2 n}\left(a_{n+1}^{(\alpha-1)}+a_{n-1}^{(\alpha-1)}\right)=\frac{1}{2}\left(a_{n+1}^{(\alpha-1)}-a_{n-1}^{(\alpha-1)}\right), \quad n \geqq 1 ;
$$

in particular $a_{0}^{*}=a_{1}^{(\alpha-1)}, a_{2 \vartheta-1}^{*}=0, v=1,2,3, \cdots$ From (5.5)

$$
a_{2 n-1}^{(\alpha-1)}-a_{2 n+1}^{(\alpha-1)}=\frac{2 \alpha}{2 n+\alpha} a_{2 n-1}^{(\alpha-1)}
$$

hence

$$
a_{2 n}^{*}=-\frac{\alpha}{2 n+\alpha} a_{2 n-1}^{(\alpha-1)}=O\left(n^{-1-\alpha}\right)
$$

and 


$$
\sum\left|a_{2 n}^{*}\right|<\infty \text {. }
$$

Let first $0<\alpha<2$; then

$$
a_{2 n}^{*}<0,
$$$$
n=1,2,3, \ldots \text {; }
$$

from (5.7) and (5.8)

$$
\sum_{1}^{\infty} a_{2 v}^{*}=-\frac{a_{1}^{(\alpha-1)}}{2}=-\frac{a_{0}^{*}}{2}
$$

hence

$$
\phi(t)=-\sum_{1}^{\infty} a_{2 v}^{*}(1-\cos 2 v t)
$$

From (5.9)

$$
a_{2}^{*}=-\frac{\alpha}{2+\alpha} a_{1}^{(\alpha-1)}=-\frac{\alpha}{2+\alpha} a_{0}^{*},
$$

hence if $2 / 3<\alpha<2$,

$$
\sum_{2}^{\infty}\left|a_{2 v}^{*}\right|=-\sum_{2}^{\infty} a_{2 v}^{*}=\frac{a_{0}^{*}}{2}+a_{2}^{*}=\frac{2-\alpha}{2 \alpha}\left|a_{2}^{*}\right|<\left|a_{2}^{*}\right| .
$$

Finally, for $\alpha \geqq 2$, from (5.9),

$$
\left|\frac{a_{2 v}^{*}}{a_{2}^{*}}\right|=\frac{2+\alpha}{2 v+\alpha} \frac{\left|a_{2 v-1}^{(\alpha-1)}\right|}{a_{1}^{(\alpha-1)}},
$$

and the inequality $\sum_{2}^{\infty}\left|a_{2 v}^{*}\right|<\left|a_{2}^{*}\right|$ follows from (5.6). Application of Theorem 4 yields the theorem:

ThForem 6'. Let $\alpha>2 / 3$,

$$
\begin{aligned}
\phi(t) & =(\sin t)^{\alpha}, & 0 \leqq t \leqq \pi, \\
\phi(-t) & =\phi(t)=\phi(t+2 \pi) . &
\end{aligned}
$$

The span of the sequence $\phi(n t), n \geqq 1$, in $C(-\pi / 2, \pi / 2)$ is the totality of all even functions vanishing for $t=0$.

For $\alpha=1$ this reduces to example 2 .

6. Application to completeness in $L_{r}$. We denote the class of $L$-integrable functions $\phi(t)$ with integrable $|\phi(t)| \cdot$ in the interval $(a, b)$ by $L_{r}(a, b)$; we assume $r \geqq 1$. The class of essentially bounded and measurable functions is denoted by $L_{\infty}$. Clearly $L_{r} \subset L_{q}$ for $r>q$; let $r^{\prime}=r /(r-1)$, and $r^{\prime}=\infty$ for $r=1 ; r$ and $r^{\prime}$ are called conjugate numbers; we have $1 / r+1 / r^{\prime}=1$. 
A sequence of functions $\phi_{n}(t) \in L_{r}(a, b), n \geqq 1$, is called complete in this space if the only function $g(t) \in L_{r^{\prime}}$ which is orthogonal to all $\phi_{n}(t)$, that is, for which

$$
\int_{a}^{b} \phi_{n}(t) \bar{g}(t) d t=0, \quad n=1,2,3, \cdots,
$$

is $g(t) \sim 0$. Here $\bar{g}(t)$ is the conjugate complex to $g(t) ; g(t) \sim 0$ means $g(t)=0$ almost everywhere.

The sequence $\phi_{n}(t) \in L_{r}(a, b)$ is called closed in this space if to any function $\psi(t) \in L_{r}(a, b)$ there exists a sequence of linear aggregates

$$
l_{n}(t)=\sum_{1}^{n} c_{v, n} \phi_{v}(t)
$$

convergent in $L_{r}$ to $\psi(t)$, that is,

$$
\int_{a}^{b}\left|\psi(t)-l_{n}(t)\right|^{r} d t \rightarrow 0 \quad \text { as } n \rightarrow \infty .
$$

Here $1 \leqq r<\infty$.

It is known that completeness and closure in $L_{r}, 1 \leqq r<\infty$, are equivalent; one implies the other.

Note that completeness in $L_{r}$ implies completeness in $L_{q}$ for any $q<r<\infty$. Furthermore with $\phi_{n}$ the sequence $c \phi_{n}, c$ a constant, is a complete system.

Consider now the equation (1.2), so that

$$
y\left(\lambda_{v} t\right)=\sum_{n=1}^{\infty} a_{n} x\left(\lambda_{n} \lambda_{v} t\right), \quad \quad v=1,2,3, \cdots ;
$$

we assume $x(t), y(t) \in L_{r}(a, b), g(t) \in L_{r^{\prime}}$. Then, if termwise integration is permitted

$$
\int_{a}^{b} y\left(\lambda_{v} t\right) \bar{g}(t) d t=\sum_{1}^{\infty} a_{n} \int_{a}^{b} x\left(\lambda_{n} \lambda_{v} t\right) \bar{g}(t) d t
$$

If we write

$$
\int_{a}^{b} x(u t) \bar{g}(t) d t=\xi(u), \quad \int_{a}^{b} y(u t) \bar{g}(t) d t=\eta(u)
$$

(6.3) becomes

$$
\eta(u)=\sum_{n=1}^{\infty} a_{n} \xi\left(\lambda_{n} u\right), \quad u=\lambda_{1}, \lambda_{2}, \lambda_{3}, \cdots .
$$

This is again an equation of the type (1.2), where now $\lambda_{1}, \lambda_{2}, \cdots$ is the set $E$ for the variable $u$. As before the sequence $\lambda_{n}$ is subject to the assumption 
that the product of any two $\lambda_{v}, \lambda_{n}$ is $a \lambda_{m}$. It is now clear that the sequence of functions $y\left(\lambda_{v} t\right)$ is complete in $L_{r}(a, b)$, if (1) the only solution of the homogenous equations

$$
\sum_{1}^{\infty} a_{n} \xi\left(\lambda_{n} u\right)=0, \quad u=\lambda_{1}, \lambda_{2}, \lambda_{3}, \cdots
$$

is

$$
\xi\left(\lambda_{n}\right)=0, \quad \text { that is, } \quad \int_{a}^{b} x\left(\lambda_{n} t\right) \bar{g}(t) d t=0,
$$$$
n=1,2, \cdots,
$$

and if (2) (6.5) implies $g(t) \sim 0$. The second condition states that the sequence $x\left(\lambda_{r} t\right)$ is complete in $L_{r}(a, b)$.

Summarizing we have the following theorem:

THEOREM 7. Assume that the sequence $x\left(\lambda_{n} t\right)$ is complete in $L_{r}(a, b)$, that $y(t) \in L_{r}(a, b)$, that (6.2) can be integrated termwise, and that (6.4) implies $(6.5)$; then the sequence $y\left(\lambda_{n} t\right)$ is complete in $L_{r}(a, b)$.

Here $1 \leqq r \leqq \infty$.

Note. The series $\sum a_{n} x\left(\lambda_{n} t\right)$ need not be convergent; it may be an orthogonal development of a function $y(t) \in L_{r}$, or associated with such a function by some summability method.

To be more specific, choose $\lambda_{n}=n$; let $x(t),-\infty<t<\infty$, be a bounded function. Assume that $x(n t), n=1,2,3, \cdots$, is a complete orthogonal system for the interval $(a, b)$. Let $2 \leqq r<\infty, y(t) \in L_{r}(a, b)$. Consider its Fourier development

$$
y(t) \sim \sum_{1}^{\infty} a_{n} x(n t) .
$$

We assume that the sequence $\left\{a_{n}\right\}$ is completely multiplicative, and that $\sum\left|a_{n}\right| r^{\prime}<\infty, r^{\prime}=r /(r-1)$. Let

$$
\xi(n)=\int_{a}^{b} x(n t) \bar{g}(t) d t,
$$

From F. Riesz' generalization of the Young-Hausdorff inequality (see, for example, [6, p. 122], [1, pp. 190-202]),

$$
\sum_{1}^{\infty}|\xi(n)| r \leqq k\left(\int_{a}^{b}|g(t)| r^{\prime} d t\right)^{-1}
$$

$k$ a constant. From (6.6)

$$
y(v t) \sim \sum_{n} a_{n} x(n v t), \quad \quad v=1,2,3, \cdots
$$


and

$$
\int_{a}^{b} y(v t) \bar{g}(t) d t=\eta(v)=\sum_{n} a_{n} \xi(\nu n) ;
$$

the series is absolutely convergent, and termwise integration is justified. We shall employ Theorem 3, where now $x(t)$ is replaced by $\xi(v)$; to the condition (4.7) corresponds

$$
\sum_{n}\left(\sum_{\delta \mid n}|\mu(\delta)|\right)\left|a_{n} \xi(v n)\right|=\sigma_{v}<\infty .
$$

But $\sum_{\delta \mid n}|\mu(\delta)| \leqq d(n)$, and $a_{n}$ being multiplicative, $\left|a_{n}\right|^{r^{\prime}}$ is multiplicative. It now follows in analogy to (4.5) that

$$
\sum\left|a_{n}\right| r^{\prime} d^{2}(n)<\infty .
$$

On the other hand, if we use Hölder's inequality,

$$
\sigma_{v} \leqq\left(\sum_{n}|\xi(v n)| r\right)^{1 / r}\left(\sum_{n}\left|a_{n}\right|^{r^{\prime}} d^{r^{\prime}}(n)\right)^{1 / r^{\prime}} ;
$$

here $r^{\prime} \leqq 2$, hence, from (6.7) and (6.9) it follows that $\sigma_{v}<\infty$. If we assume now $\eta(v)=0, v=1,2, \cdots$, Theorem 3 yields $\xi(n)=0, n=1,2, \cdots$; but the sequence $x(n t)$ is complete, hence $g(t) \sim 0$. Summarizing we have proved:

THeOREM 8. Let $x(t)$ be bounded, $-\infty<t<\infty, x(n t), n=1,2, \cdots$, an orthogonal sequence in $(a, b)$, complete in $L_{r}(a, b), r \geqq 2$. If $y(t) \in L_{r}$, if its Fourier coefficients $a_{n}$ satisfy (4.1), and $\sum\left|a_{n}\right| r^{\prime}<\infty$, then the sequence $y(n t)$, $n=1,2, \cdots$, is complete in $L_{r}(a, b)$.

\section{Application to special cases.}

1. Let $x(t)=-\sin 2 \pi t$, then the sequence $x(n t), n \geqq 1$, is orthogonal and complete in $L_{r}(0,1 / 2)$ for all $r>1$. Let

$$
y(t)=-\sum_{1}^{\infty} \frac{\sin 2 v \pi t}{v}=\sum_{1}^{\infty} \frac{x(v t)}{v},
$$

so that $a_{v}=v^{-1}, a_{n} a_{v}=a_{n v}$. It is known that $y(t)=\pi(t-[t j-1 / 2)$ for $t>0$, not an integer. Now $y(t) \in L_{r}$ for all $r>1, \sum v^{r^{\prime}}<\infty$ for all $r^{\prime}>1$; application of Theorem 8 yields:

THEOREM 9. The sequence $n t-[n t]-1 / 2$ is complete in $L_{r}(0,1 / 2)$ for all $r>1\left(^{(3)}\right.$.

The functions $\sin \pi t, \sin 3 \pi t, \cdots$, being orthogonal to the sequence $x(n t)$ in $(0,1)$, are also orthogonal to the sequence $y(n t)$; we note that N. P. Roma-

(3) For $r=2$ see Wintner [10]. 
noff [7] states that the system $1, y(n t), u(n t)$, where $u(t)=t^{2}-t+1 / 6,0 \leqq t \leqq 1$, and of period 1 , is complete in $L_{2}(0,1)$.

Utilizing the uniform convergence of (7.2), it is seen that the span of the sequence $\sin 2 n \pi t$ in $C(0,1)$ is included in the span of the sequence $y(n t)$. Furthermore

$$
\pi^{2} u(t)=\sum_{1}^{\infty} v^{-2} \cos 2 v \pi t, \quad 0 \leqq t \leqq 1,
$$

hence, by Theorem 3,

$$
\cos 2 \pi t=\pi^{2} \sum_{1}^{\infty} \mu(v) v^{-2} u(v t)
$$

and the series is evidently uniformly convergent. It follows that the span of the sequence $\cos 2 n \pi t, n \geqq 1$, is identical with the span of the sequence $u(n t)$ in $C(0,1)$. This yields Romanoff's result not only for $L_{2}$ but for $C(0,1)$. For $L_{r}(0,1), r>1$, the corresponding proof is elementary.

2. Let $x(t)=(4 / \pi)$ sin $\pi t$; the sequence $x(n t)$ is orthogonal and complete in $L_{r}(0,1)$ for all $r$; let

$$
y(t)=\frac{4}{\pi} \sum_{1}^{\infty} \frac{\sin (2 v-1) \pi t}{2 v-1},
$$

so that $a_{n}=0$ if $n$ even, $a_{n}=1 / n$ if $n$ odd. We have again $a_{n} a_{v}=a_{n v}$. Furthermore $y(t)=\operatorname{sgn} \sin \pi t \in L_{r}$ for all $r>1, \sum(2 v-1)^{-r^{\prime}}<\infty$ for all $r^{\prime}>1$. From Theorem 8 it now follows:

THEOREM 10. The sequence $\operatorname{sgn} \sin n \pi t, n=1,2, \cdots$, is complete in $L_{r}(0,1)$ for all $r>1$.

The sequence $y\left(2^{v} t\right), v=0,1,2, \cdots$, is the well known Rademacher set, which is orthogonal, but incomplete in $L_{2}(0,1)$.

2. Let $x(t)=\cos 2 \pi t$; the sequence $1, x(n t)$ is complete and orthogonal in $L_{r}(0,1 / 2), r>1$. Consider

$$
y(t)=\sum_{1}^{\infty} v^{-1} x(v t)=\sum_{1}^{\infty} v^{-1} \cos 2 v \pi t=-\log |2 \sin \pi t| .
$$

We now get:

THEOREM $10^{\prime}$. The sequence $1, \log |2 \sin n \pi t|$ is complete in $L_{r}(0,1 / 2)$ for all $r>1$.

A generalization of (7.1) is

$$
y(t) \sim \sum_{1}^{\infty} \frac{\sin 2 v \pi t}{v^{\alpha}}, \quad 1 / 2<\alpha<1 .
$$


We have $\sum_{1}^{\infty} v^{-\alpha r^{\prime}}<\infty$ for $\alpha r^{\prime}>1$, or $r<1 /(1-\alpha)$; hence, by the YoungHausdorff inequalities (see $\left[11\right.$, p. 190]) $y(t) \in L_{r}$ for $r<1 /(1-\alpha)$, and by Theorem 8 the sequence $\{y(n t)\}$ is complete in $L_{r}(0,1 / 2)$ for $r<1 /(1-\alpha)$. A similar result holds for the corresponding cosine series. For $r=2$ see Wintner [10].

P. Erdös called my attention to two interesting papers by $H$. Davenport [3]. It is proved in the second paper that the series

$$
\sum \frac{\mu(n)}{n}\{n t\}=-\frac{\sin 2 \pi t}{\pi},
$$

where

$$
\begin{array}{ll}
\{t\}=t-[t]-1 / 2, & \text { if } t \neq[t], \\
\{t\}=0, & \text { if } t=[t],
\end{array}
$$

is uniformly convergent. Hence any function belonging to the span of the sequence $\sin 2 n \pi t$ in $C(0,1 / 2)$ also belongs to the span of the sequence $\{n t\}$ $n=1,2,3, \ldots$. Thus we have the theorem:

THEOREM 11. Any function continuous in the closed interval [0, 1/2], and vanishing at the end points, can be approximated uniformly by linear aggregates of the functions $\{n t\}$.

It should be noted that Davenport, in proving the uniform convergence of the series (7.2), uses a method based on Vinogradov's recent work on the theory of primes. This is only natural when we observe that the uniform convergence of (7.2) implies the prime number theorem. In fact, if we write

$$
s_{n}(t)=\sum_{1}^{n} \frac{\mu(v)}{v}\{v t\}, \quad n=1,2,3, \cdots,
$$

it follows from uniform convergence that $s_{n}\left(n^{-2}\right) \rightarrow 0$, or $n^{-2} \sum_{1}^{n} \mu(v)$ $-2^{-1} \sum_{1}^{n} v^{-1} \mu(v) \rightarrow 0$ as $n \rightarrow \infty$. Hence $\sum_{1}^{n} v^{-1} \mu(v) \rightarrow 0$, and this is known to be equivalent to the prime number theorem.

We discuss here the limiting case $\sum_{2}^{\infty}\left|a_{n}\right|=1$ of Theorem 7 for the special case of sine or cosine series and for completeness in $L_{\infty}$.

Let $g(t) \in L_{1}$; then

$$
\int_{0}^{\pi} y(v t) \bar{g}(t) d t=\sum_{n=1}^{\infty} a_{n} \int_{0}^{\pi} \sin v n t \bar{g}(t) d t .
$$

If

$$
\int_{0}^{\pi} y(v t) \bar{g}(t) d t=0 \quad \text { for } v=1,2,3
$$


then

$$
\sum_{n=1}^{\infty} a_{n} c_{v n}=0, \quad \quad v=1,2,3, \cdots
$$

where

$$
c_{k}=\int_{0}^{\pi} \sin k t \bar{g}(t) d t \rightarrow 0 \quad \text { as } k \rightarrow \infty .
$$

Assume that not all $c_{k}$ are equal to 0 ; let $\max _{n \geq k}\left|c_{n}\right|=\gamma_{k}$, then $\gamma_{k} \downarrow 0$, hence there is a $k$ so that $\gamma_{k}>\gamma_{v}$ for $v>k$. For such a $k$ we have

$$
\left|c_{k}\right|>\left|c_{v}\right|, \quad \text { for } v>k \text {; }
$$

now from (7.3)

$$
c_{k}=-\sum_{n=2}^{\infty} a_{n} c_{k n}
$$

hence

$$
\left|c_{k}\right|<\left|c_{k}\right| \sum_{2}^{\infty}\left|a_{n}\right| \leqq\left|c_{k}\right|
$$

which is a contradiction; thus $c_{n}=0$ for $n=1,2,3, \cdots$. We have proved the theorem :

THEOREM 12. If

$$
y(t)=\sin t+\sum_{2}^{\infty} a_{n} \sin n t, \quad \sum_{2}^{\infty}\left|a_{n}\right| \leqq 1,
$$

then the sequence $\{y(n t)\}$ is complete in $L_{\infty}(0, \pi)$.

A similar result holds for cosine series. In particular the sequence of Theorem 6 is complete in $L_{\infty}(0, \pi)$ for $\alpha=1 / 3$, and the sequence of Theorem $\sigma^{\prime}$ is complete in $L_{\infty}(0, \pi / 2)$ for $\alpha=2 / 3$.

8. Closure and completeness in $C(0,1)$. We say that a sequence $\phi_{n}(t), n$ $=1,2, \cdots$, of continuous functions is closed in $C(0,1)$, or a base for the interval $(0,1)$, if any function, continuous in the closed interval $(0,1)$, can be approximated uniformly in $(0,1)$ by linear aggregates of the functions $\phi_{n}(t)$. The sequence is called complete in $C(0,1)$ if for any function $\psi(t)$ of bounded variation the infinitely many equations

$$
\int_{0}^{1} \Phi_{n}(t) d \psi(t)=0, \quad n=1,2,3, \cdots
$$

imply $\psi(t)=\psi(1)=\psi(0)$, except at an enumerable set of points $t$. Here $\Phi_{n}$ is 
the conjugate complex of $\phi_{n}(t)$. It is known that closure and completeness in $C(0,1)$ are equivalent (see, for example $[1$, p. 73$])$. Let

$$
f_{n}(t)=\phi(t) \phi_{n}(t)
$$

where $\phi(t)$ is continuous in the closed interval $(0,1)$. Assume that the sequence $\left\{\phi_{n}(t)\right\}$ is closed in $C(0,1)$; we shall investigate the closure properties of the sequence (8.2).

Normalizing $\psi(t)$ by $\psi(0)=0, \psi(t)=\{\psi(t-0)+\psi(t+0)\} / 2$ for $0<t<1$, the condition of closure is that (8.1) shculd imply $\psi(t)=0$ for $0 \leqq t \leqq 1$.

We first give three lemmas.

LEMma 1. Let $f(x), \phi(x)$ be continuous, $\psi(x)$ of bounded variation in $[0,1]$; let

$$
\eta(x)=\int_{0}^{x} \phi(t) d \psi(t)
$$

Then

$$
\int_{0}^{1} f(x) \phi(x) d \psi(x)=\int_{0}^{1} f(x) d \eta(x) .
$$

Cf. D. V. Widder, The Laplace transform, Princeton, 1946; in particular Theorem 6b, p. 12.

LEMma 2. If $\psi(t)$ is normalized, then $\eta(t)$ is normalized.

See Widder, Theorem $8 b$, p. 14.

Lemma 3. Assume that $\phi(t) \neq 0$ and has a continuous derivative for $0<t<1$, that $\phi(t)$ is continuous in $[0,1]$, and that $\phi(0) \neq \phi(1)$. Let $\psi(t)$ be normalized; if

$$
\eta(x)=\int_{0}^{x} \phi(t) d \psi(t)=0 \quad \text { for } 0 \leqq x \leqq 1,
$$

and if $\psi(0)=\psi(1)=0$, then $\psi(t)=0$ for $0 \leqq t \leqq 1$.

We have

$$
\begin{aligned}
\eta(x) & =\phi(x) \psi(x)-\int_{0}^{x} \psi(t) d \phi(t) \\
& =\phi(x) \psi(x)-\int_{0}^{x} \psi(t) \phi^{\prime}(t) d t=0,
\end{aligned}
$$

or

$$
\phi(x) \psi(x)=\int_{0}^{x} \psi(t) \phi^{\prime}(t) d t, \quad 0 \leqq x \leqq 1
$$


The right side is continuous in $(0,1)$, hence $\psi(x)$ is continuous in $(0,1)$; it now follows that the right side is differentiable in $(0,1)$, hence $\psi(x)$ is differentiable in $(0,1)$, and

$$
\phi^{\prime}(x) \psi(x)+\phi(x) \psi^{\prime}(x)=\psi(x) \phi^{\prime}(x), \quad 0<x<1,
$$

or

$$
\phi(x) \psi^{\prime}(x)=0, \quad 0<x<1
$$

It follows that

$$
\psi^{\prime}(x)=0, \quad 0<x<1,
$$

or

$$
\psi(x)=c, \text { a constant } \quad 0<x<1 .
$$

Now from $(8.5)$

$$
\phi(x) \psi(x)=c\{\phi(x)-\phi(0)\}, \quad 0 \leqq x \leqq 1,
$$

and for $x=1$

$$
\phi(1) \psi(1)=0=c\{\phi(1)-\phi(0)\} ;
$$

hence $c=0$. This proves Lemma 3 .

Remark. If $\phi(0)=\phi(1)$, while the other assumptions of Lemma 3 hold, and if $g(t)$ is a continuous function for which $\int_{0}^{1} \bar{g}(t) d \psi(t)=0, g(0) \neq g(1)$, then again $\psi(t) \equiv 0$. We have as before $\psi(t)=c$ for $0<t<1$; hence

$$
\int_{0}^{1} \bar{g}(t) d \psi(t)=c\{\bar{g}(0)-\bar{g}(1)\}=0,
$$

and $c=0$.

Assume now that $\psi(t)$ is normalized, that

$$
\int_{0}^{1} \Phi_{n}(t) \phi(t) d \psi(t)=0, \quad n=1,2,3, \cdots,
$$

and

$$
\int_{0}^{1} d \psi(t)=\psi(1)-\psi(0)=0
$$

Thus $\psi(1)=\psi(0)=0$. By Lemma 1, (8.6) can be written in the form

$$
\int_{0}^{1} \Phi_{n}(t) d \eta(t)=0, \quad n=1,2,3, \cdots
$$

where 


$$
\eta(x)=\int_{0}^{x} \Phi(t) d \psi(t)
$$

by Lemma $2, \eta(x)$ is a normalized function of bounded variation. If the sequence is complete in $C(0,1)$, then $\left(8.6^{\prime}\right)$ implies $\eta(t) \equiv 0$. Employing Lemma 3 and the additional remark, we now get the following theorem:

TheOREM 13. Assume that the sequence $\left\{\phi_{n}(t)\right\}$ is closed in $C(0,1)$, that $\phi(t) \neq 0$ and has a continuous derivative in $(0,1)$, that $\phi(t)$ is continuous in $[0,1]$, and that $\phi(0) \neq \phi(1)$. Then the sequence

$$
1, \phi(t) \phi_{n}(t), \quad \quad n=1,2,3, \cdots,
$$

is closed in $C(0,1)$. If $\phi(0)=\phi(1)$, if $g(t)$ is continuous and $g(0) \neq g(1)$, then the sequence

$$
1, g(t), \phi(t) \phi_{n}(t), \quad n=1,2,3, \cdots
$$

is closed in $C(0,1)(4)$.

Special cases: Let

$$
\begin{aligned}
\phi_{1}(t) & =1, \quad \phi_{n}(t)=t^{\gamma_{n}}, \quad n=2,3, \cdots, \\
R \gamma_{n}>0, \quad \gamma_{v} & \neq \gamma_{k}, \quad \sum \frac{R \gamma_{v}}{1+\left|\gamma_{v}\right|^{2}}=\infty .
\end{aligned}
$$

It is known (see [8]), that under the assumptions (8.8) the sequence (8.7) is closed in $C(0,1)$. Theorem 13 now yields:

TheOREM 13'. If (8.8) holds, if $\phi(t)$ is continuous in $[0,1], \phi(t) \neq 0$, and $\phi^{\prime}(t)$ continuous in $(0,1)$ and if $\phi(0) \neq \phi(1)$ then the sequence

$$
1, \phi(t), \phi(t) t^{\gamma_{n}}, \quad n=2,3, \cdots,
$$

is closed in $C(0,1)$.

In particular let $\phi(t)=(1-t)^{\delta} h(t), \delta \geqq 0, h(0) \neq 0$; assume that $h(t)$ is continuous in $[0,1]$, and has a continuous derivative in $(0,1)$. If $(8.8)$ holds, then the sequence

$$
\text { 1, } h(t)(1-t)^{\delta}, \quad h(t)(1-t)^{\delta} t^{\gamma_{n},} \quad n=2,3, \cdots,
$$

is closed in $C(0,1)$. Thus the sequence

$$
1, \quad h(t)(1-t)^{\delta}, \quad h(t)(1-t)^{\delta} t^{n-\alpha}, \quad \alpha \leqq 1 ; n=2,3, \cdots,
$$

is closed in $C(0,1)$.

Let $\phi(t)=t h(t)(\log (1 /(1-t)))^{-1}$, where $h(t)$ satisfies the conditions stated

(4) For a more general result with a different proof see a forthcoming paper by J. Korevaar in the Duke Mathematical Journal. 
above; the sequence

$$
1, h(t) t^{n}\left(\log \frac{1}{1-t}\right)^{-1}, \quad n=1,2,3, \cdots
$$

is closed in $C(0,1)$.

Let $\phi(t)=t(\log (1 / t))^{\delta}, \delta>0$, thus $\phi(0)=\phi(1)=0$; if we put $g(t)=t$, $\phi_{n}(t)=t^{n-1}, n=1,2, \cdots$, it follows from Theorem 13 that the sequence

$$
1, t, t^{n}\left(\log \frac{1}{t}\right)^{\delta}
$$$$
\delta>0, n=1,2, \cdots,
$$

is closed in $C(0,1)$.

9. Application of the preceding results. Let

$$
k_{\beta}(t)=\sum_{1}^{\infty} n^{\beta} t^{n}, \quad 0 \leqq t<1,-1<\beta \leqq 2 .
$$

If we put $t=e^{-s}, s>0$, Theorem 3 yields

$$
e^{-s}=\sum_{1}^{\infty} \mu(n) n^{\beta} k_{\beta}\left(e^{-n s}\right),
$$

or

$$
t=\sum_{1}^{\infty} \mu(n) n^{\beta} k_{\beta}\left(t^{n}\right)
$$

If was proved elsewhere $[5, \mathrm{II}]$, that for $-1<\beta \leqq 2$ the series

$$
\sum_{1}^{\infty} \mu(n) n^{\beta}(1-t)^{\beta+1} k_{\beta}\left(t^{n}\right)=t(1-t)^{\beta+1}
$$

converges boundedly in $0 \leqq t<1$, and the partial sums are bounded by $(1-t)^{\beta+1} k_{\beta}(t)$ for all $n$; we have concluded in $[5$, II] that for any function $\psi(t)$ of bounded variation

$$
\sum_{1}^{\infty} \mu(n) n^{\beta} \int_{0}^{1}(1-t)^{\beta+1} k_{\beta}\left(t^{n}\right) d \psi=\int_{0}^{1} t(1-t)^{\beta+1} d \psi .
$$

J. Korevaar pointed out to me that this conclusion is incorrect, unless we use the convergence of $(9.1)$ in the closed interval to a continuous function. We have mentioned however that this can be proved on using the prime number theorem. In fact defining $(1-t)^{\beta+1} k_{\beta}(t)$ for $t=1$ by

$$
\lim _{t \rightarrow 1}(1-t)^{\beta+1} k_{\beta}(t)=\Gamma(\beta+1),
$$

we get 


$$
\lim _{t \rightarrow 1}(1-t)^{\beta+1} k_{\beta}\left(t^{n}\right)=\frac{\Gamma(\beta+1)}{n^{\beta+1}},
$$

and, for $t=1$, (9.1) becomes $\sum_{1}^{\infty} n^{-1} \mu(n)=0$, which is equivalent to the prime number theorem.

More generally, we shall employ:

Lemma 4. Let $\alpha \leqq 1,-1<\beta \leqq 2$; the series

$$
\sum_{1}^{\infty} \mu(n) n^{\beta} t^{-\alpha}(1-t)^{\beta+1} \dot{k}_{\beta}\left(t^{n}\right)=t^{1-\alpha}(1-t)^{\beta+1}
$$

converges boundedly to a continuous function in the closed interval $0 \leqq t \leqq 1$.

The partial sums of (9.2) are bounded by

$$
t^{-\dot{\alpha}}(1-t)^{\beta+1} k_{\beta}(t) \leqq t^{-1}(1-t)^{\beta+1} k_{\beta}(t)=Q_{\beta}(t),
$$

say. It was shown [5, II, Lemma 5.1] that for $\beta \geqq 1,0<t<1, Q_{\beta}(t)$ is an increasing function of $t$; hence

$$
Q_{\beta}(t) \leqq Q_{\beta}(1)=\Gamma(\beta+1) .
$$

Furthermore

$$
t^{-1} k_{\beta}(t)=\int_{0}^{1}(t u)^{-1} k_{\beta+\mathrm{i}}(t u) d u
$$

hence, for $\beta \geqq 0$

$$
t^{-1} k_{\beta}(t) \leqq \Gamma(\beta+2) \int_{0}^{1}(1-t u)^{-\beta-2} d u=\Gamma(\beta+1) t^{-1}\left\{(1-t)^{-\beta-1}-1\right\},
$$

or

$$
Q_{\beta}(t) \leqq \Gamma(\beta+1) t^{-1}\left\{1-(1-t)^{\beta+1}\right\}, \quad \beta \geqq 0 .
$$

But $t^{-1}\left\{1-(1-t)^{\beta+1}\right\} \uparrow 1$ as $t \uparrow 1$, hence

$$
Q_{\beta}(t) \leqq \Gamma(\beta+1), \quad \beta \geqq 0 .
$$

Repeating this process we find easily $Q_{\beta}(t) \leqq \Gamma(\beta+2), \beta>-1$. This proves the lemma.

It follows that the series

$$
\sum_{1}^{\infty} \mu(n) n^{\beta} t^{-\alpha}(1-t)^{\beta+1} k_{\beta}\left(t^{v n}\right)=t^{v-\alpha}(1-t)^{\beta+1}, \quad v \geqq 1 ; 0 \leqq t \leqq 1,
$$

converges boundedly. Assume that $h(t) \neq 0$ and has a continuous derivative in $(0,1)$; then termwise integration yields 


$$
\sum_{1}^{\infty} \mu(n) n^{\beta} \int_{0}^{1} t^{\alpha}(1-t)^{\beta+1} h(t) k_{\beta}\left(t^{v n}\right) d \psi=\int_{0}^{1} t^{v-\alpha}(1-t)^{\beta+1} h(t) d \psi .
$$

Assume that

$$
\int_{0}^{1} t^{\alpha}(1-t)^{\beta+1} h(t) k_{\beta}\left(t^{n}\right) d \psi=0, \quad n=1,2, \cdots,
$$

then

$$
\int_{0}^{1} t^{v-\alpha}(1-t)^{\beta+1} h(t) d \psi=0, \quad v=1,2, \cdots .
$$

We further assume that $\psi(t)$ is normalized,

$$
\int_{0}^{1} d \psi=0, \quad \int_{0}^{1}(1-t)^{\beta+1} h(t) d \psi=0
$$

and that $h(0) \neq 0$. The sequence

$$
1,(1-t)^{\beta+1} h(t), t^{v-\alpha}(1-t)^{\beta+1} h(t), \quad v=1,2, \cdots,
$$

being complete in $C(0,1)$ (see (8.9)), it follows that $\psi(t) \equiv 0$. We have thus proved:

THEOREM 14. Assume that $h(t) \neq 0$ and has a continuous derivative in $(0,1)$, that $h(t)$ is continuous in $[0,1]$ and that $h(0) \neq 0$. Let $\alpha \leqq 1,-1<\beta \leqq 2$; then the sequence

$$
\text { 1, }(1-t)^{\beta+1} h(t), t^{-\alpha}(1-t)^{\beta+1} h(t) k_{\beta}\left(t^{n}\right), \quad n=1,2, \cdots,
$$

is complete in $C(0,1)$.

For $h(t)=(1-t)^{\rho}, \rho \geqq 0, \alpha=0$ (see [5, I, II]); there the second term was omitted by an oversight. For $\rho>0$ this is clearly not permissible as then all linear combinations in the sequence take on equal values at 0 and 1 . This was pointed out to me by J. Korevaar.

For $\alpha=1$ the second of the conditions (9.4) is included in (9.3). We thus get the following theorem.

THEOREM 14*. If $\beta$ and $h(t)$ satisfy the conditions of Theorem 14, then the sequence

$$
1, t^{-1}(1-t)^{\beta+1} h(t) k_{\beta}\left(t^{n}\right), \quad n=1,2,3, \cdots
$$

is complete in $C(0,1)$.

In particular $(\beta=0$ and $\beta=1)$ each of the sequences $1,\left((1-t) t^{n-1} /\left(1-t^{n}\right)\right)$ . $h(t) ; 1,\left((1-t)^{2} t^{n-1} /\left(1-t^{n}\right)^{2}\right) h(t), n \geqq 1$, is complete in $C(0,1)$.

While in [5] complex function theory was employed, the proof given here 
presents a simplification. However, the complex method permits some generalizations. If we put

$$
h(t)=t^{\alpha}\left(\frac{\log 1 / t}{1-t}\right)^{\beta+1}, \quad 0<\alpha \leqq 1
$$

(9.3) becomes

$$
\int_{0}^{1} t^{v}\left(\log \frac{1}{t}\right)^{\beta+1} d \psi(t)=0, \quad v=1,2,3, \cdots
$$

Now $h(0)=0$; assume that

$$
\int_{0}^{1} d \psi(t)=0, \quad \int_{0}^{1} t d \psi(t)=0 .
$$

The sequence

$$
1, t, t^{v}(\log (1 / t))^{\beta+1}, \quad v=1,2,3, \cdots,
$$

is complete in $C(0,1)$ (see $(8.11)$ ); this yields:

TheOREM $14^{\prime}$. Let $-1<\beta \leqq 2$; the sequence

$$
1 ; t,(\log (1 / t))^{\beta+1} k_{\beta}\left(t^{n}\right), \quad n=1,2,3, \cdots,
$$

is complete in $C(0,1)$.

Putting $\beta=0$ or $\beta=1$, and $t=e^{-s}$, we get the corollary. Each of the sequences

$$
\begin{aligned}
1, e^{-s}, \frac{s}{e^{n s}-1}, & n=1,2,3, \cdots, \\
1, e^{-s}, \frac{s^{2}}{\left(e^{n s}-1\right)^{2}}, & n=1,2,3, \cdots,
\end{aligned}
$$

is complete in $C(0, \infty)$.

The extremal case $\beta=-1$ of Theorem 14 deserves separate treatment. Now

$$
\sum_{1}^{\infty} n^{-1} t^{n}=\log \frac{1}{1-t}, \quad 0 \leqq t<1
$$

and Thecrem 3 yields

$$
t=\sum_{1}^{\infty} \frac{\mu(n)}{n} \log \frac{1}{1-t^{n}} .
$$

We have 


$$
s_{n}(t) \equiv \sum_{1}^{n} \frac{\mu(v)}{v} \log \frac{1}{1-t^{0}}=\sum_{1}^{n-1} \mu^{*}(v) \log \frac{1-t^{v+1}}{1-t^{0}}-\mu^{*}(n) \log \left(1-t^{n}\right),
$$

where

$$
\mu^{*}(n)=\sum_{1}^{n} \frac{\mu(v)}{v}, \quad\left|\mu^{*}(n)\right| \leqq 1 ;
$$

furthermore

$$
\log \frac{1-t^{v+1}}{1-t^{v}}>0
$$

hence

$$
\left|s_{n}(t)\right| \leqq-\log (1-t), \quad 0 \leqq t<1 .
$$

If $h(t)$ is continuous in $[0,1]$, then the series

$$
\sum_{1}^{\infty} \frac{\mu(n)}{n} \frac{\log \left(1-t^{n}\right)}{\log (1-t)} h(t)=-\frac{t h(t)}{\log (1-t)}
$$

is boundedly convergent, and

$$
\sum_{1}^{\infty} \frac{\mu(n)}{n} \frac{\log \left(1-t^{v n}\right)}{\log (1-t)} h(t)=-\frac{t^{v} h(t)}{\log (1-t)}
$$

is boundedly convergent to a continuous function; here $v=1,2,3, \cdots$. Now termwise integration yields

$$
\sum_{1}^{\infty} \frac{\mu(n)}{n} \int_{0}^{1} \frac{\log \left(1-t^{\nu n}\right)}{\log (1-t)} h(t) d \psi=-\int_{0}^{1} \frac{t^{v} h(t)}{\log (1-t)} d \psi
$$

assume that $\int_{0}^{1} d \psi(t)=0$, and

$$
\int_{0}^{1} \frac{\log \left(1-t^{n}\right)}{\log (1-t)} h(t) d \psi(t)=0, \quad n=1,2,3, \cdots ;
$$

then

$$
\int_{0}^{1} \frac{t^{v} h(t)}{\log (1-t)} d \psi(t)=0, \quad v=1,2,3, \cdots .
$$

If $h(t)$ satisfies the conditions of Thecrem 14 , then the sequence

$$
1, \frac{t^{v} h(t)}{\log (1-t)}, \quad \quad v=1,2,3, \cdots,
$$

is complete in $C(0,1)$ (see $(8.10))$, hence the result: 
THEOREM 15. If $h(t)$ satisfies the conditions of Theorem 14 , then the sequence

$$
1, h(t), \frac{\log \left(1-t^{n}\right)}{\log (1-t)}, \quad n=1,2,3, \cdots
$$

is complete in $C(0,1)$.

\section{REFERENCFS}

1. S. Banach, Theorie des operations lineaires, Warsaw, 1932.

2. D. G. Bourgin, On certain sequences of functions, Proc. Nat. Acad. Sci. U.S.A. vol. 32 (1946).

3. H. Davenport, On some infinite series involving arithmetical functions, Quart. J. Math. Oxford Ser. vol. 8 (1937) pp. 8-13, 313-320.

4. E. Hille, The inversion problem of Möbius, Duke Math. J. vol. 3 (1937) pp. 549-568.

5. E. Hille and O. Szász, On the completeness of Lambert functions. I, Bull. Amer. Math. Soc. vol. 42 (1936) pp. 411-418; II, Ann. of Math. vol. 37 (1936) pp. 801-815.

6. F. Riesz, Über eine Verallgemeinerung der Parsevalschen Formel, Math. Zeit. vol. 18 (1923) pp. 117-124.

7. N. P. Romanoff, On a special orthonormal system, C. R. (Doklady) Acad. Sci. URSS. N.S. vol. 40 (1943) pp. 257-258.

8. O. Szász, Über die Approximation stetiger Funktionen durch lineare Aggregate von Potenzen, Math. Ann. vol. 77 (1916) pp. 482-496.

9. Lectures on the analytic theory of numbers, University of Cincinnati, 1938-1939.

10. A. Wintner, Diophantine approximations and Hilbert's space, Amer. J. Math. vol. 66 (1944) pp. 564-578.

11. A. Zygmund, Trigonometrical series, Warsaw, 1935.

\section{RECENT RELEVANT LITERATURE}

D. G. Bourgin, A class of sequences of functions, Trans. Amer. Math. Soc. vol. 60 (1946) pp. 478-518.

A. Wintner, A solution theory of the Möbius transform, Amer. J. Math. vol. 68 (1946) pp. 321-338.

UNiversity of CincinNati,

Cincinnati, Ohio. 